FEDSM2 $005-77477$

\title{
CAVITATION EFFECTS ON FLUID STRUCTURE INTERACTION IN THE CASE OF A 2D HYDROFOIL
}

\author{
Philippe Ausoni Mohamed Farhat François Avellan \\ Ecole Polytechnique Federale de Lausanne \\ Laboratory for hydraulic machines \\ Av. Cour 33bis, 1007 Lausanne \\ Switzerland \\ Xavier Escaler Eduard Egusquiza \\ Universitat Politecnica de Catalunya \\ Av. Diagonal 647, 08028 Barcelona \\ Spain
}

\begin{abstract}
In the present study, we have carried out an experimental investigation on the fluid-structure interaction caused by Karman vortices in the wake of a truncated 2D hydrofoil. The instrumentation involves a high frequency accelerometer and high speed visualisation. The mechanical response of the hydrofoil to the hydrodynamic excitation is monitored with the help of a portable digital vibrometer. Moreover, a specific optical device is developed to investigate the dynamic of the cavitating wake. The survey of the generation frequency of the Karman vortices with respect to the flow velocity reveals a Strouhal behaviour and three resonances of the hydrofoil. Out of hydro-elastic coupling conditions, the observation of the vortex structures reveals a strong $3 \mathrm{D}$ pattern despite the fact that the hydrofoil is $2 \mathrm{D}$. The maximum fluid-structure interaction occurs for the torsional mode where lock-in is observed for upstream velocities ranging from 11 to $13 \mathrm{~m} / \mathrm{s}$. In this case, the vortices exhibit a 2D structure. The cavitation occurrence within the core of Karman vortices leads to a significant increase of their generation frequency. We have observed that hydrofoil resonance may be whether avoided or triggered by cavitation development. The study of the Karman vortices dynamic reveals that their advection velocity increases (4\%) with the development of the wake cavitation meanwhile their streamwise spacing decreases.
\end{abstract}

\section{INTRODUCTION}

Placed in a fluid stream, bodies generate separated flow over a substantial proportion of their surface. The boundary layers detach from each side of the structure and form two shear layers, which roll into the near wake, where they fold on each other and coalesce into discrete swirling vortices [7]. The resulted von Karman vortex street and its interactions with the structures have been the issue of research for more than a century (Rockwell [3] and Williamson [4] for recent reviews). The vortex shedding frequency is found to be proportional to the upstream velocity through the Strouhal law. As the flow velocity is increased or decreased so that the shedding frequency approaches one of the natural frequencies of the combined fluid-structure system, a resonance (hydro-elastic coupling) occurs and significant increases of noise and vibration level are observed. According to hydro-elastic coupling and resulting amplitude of vibration, the shedding frequency can lock onto the natural frequency on a specific range of upstream velocity, defining the lock-in phenomenon.

The presence of cavitating liquid in the wake of the body allows for flow visualisation of the vortex street. The bubbles initiate and persist in the minimum pressure centres of the individual shed vortices. Cavitation forming in the turbulent wake of bluff bodies has been investigated by researchers, including Dupont et al. [2], Young and Holl [5] and Belahadji et al.[6]. Notable influences of the cavitation on the dynamic of the vortex street have been observed. Developed wake cavitation will typically increase the shedding frequency of the von Karman vortices up to $25 \%$ and will actively alter the dynamics of the turbulent wake.

As matters stand, these effects have to be investigated in the case of von Karman vortices generated in the wake of a truncated 2D hydrofoil. This case study is of practical interest in many branches of engineering. Moreover, no detailed study has been carried out so far in order to distinguish the cavitation effects in or out of hydro-elastic coupling conditions. This paper will describe the experimental facilities and will show how the cavitation development in the core of Karman vortices acts on the fluid structure interaction. 


\section{NOMENCLATURE}

A

Streamwise spacing of vortices in the same row

Cross-stream spacing of the two rows of vortices

B

Hydrofoil chord length and span

$c, l$ Distance from the trailing edge in the free stream direction

d

$f_{s} \quad$ Shedding frequency of Karman vortices

$h \quad$ Hydrofoil trailing edge thickness

$p_{\text {inlet }} \quad$ Reference pressure at the test section inlet

$p_{v} \quad$ Vapour pressure

$\mathrm{Re}_{\text {chord }}$ Reynolds number: $\mathrm{Re}_{\text {chord }}=U_{r e f} c / v$

St Strouhal number: $S t=f_{s} h / U_{\text {ref }}$

$U_{\text {ref }} \quad$ Reference velocity at the test section inlet

$U_{\text {Karman }}$ Advection velocity of the vortices

$U^{*} \quad$ Reduced velocity: $U^{*}=U_{\text {karman }} / U_{r e f}$

$\alpha \quad$ Incidence angle of the hydrofoil

Cavitation index:

$$
\sigma=\frac{p_{\text {inlet }}-p_{v}}{\frac{1}{2} \rho U_{r e f}^{2}}
$$

Incipient cavitation index

$\begin{array}{ll}\sigma_{\mathrm{i}} & \text { Incipient cavitation } \\ v & \text { Kinematic viscosity }\end{array}$

\section{(ex}

\section{EXPERIMENTAL SETUP}

\section{Cavitation tunnel and hydrofoil}

The EPFL high-speed cavitation tunnel, Avellan et al.[1] (Figure $1 \mathrm{Up}$ ), is a closed loop with a test section of $150 \times 150 \times 750 \mathrm{~mm}$. The operating flow parameters are the upstream velocity $U_{r e f}$ and the pressure at the inlet of the test section $p_{\text {inlet }}$, which together define the cavitation index $\sigma$. The variability of the static pressure in the test section permits studies over a wide range of cavitation degree.

The experimental 2D hydrofoil (Figure 1 Down) is a Naca0009 made of stainless steel. The hydrofoil is truncated at $90 \%$ of its original chord length. The resulting trailing edge thickness $h$ is $3.22 \mathrm{~mm}$ and its chord $c$ and span $l$ lengths are $100 \mathrm{~mm}$ and $150 \mathrm{~mm}$ respectively. The boundary conditions of the hydrofoil in the test section are a perfect embedding on one side and a pivot embedding on the other. The incidence angle of the hydrofoil $\alpha$ is fixed at $0^{\circ}$ for all experiments.

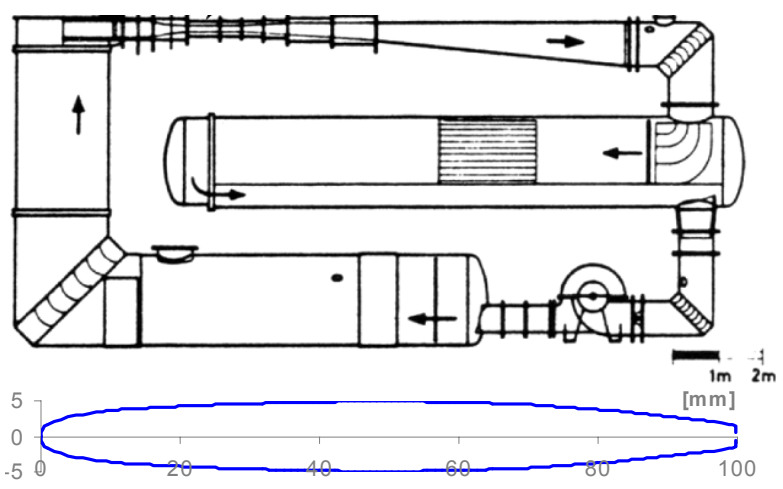

Figure $1:$ (Up) EPFL high speed cavitation tunnel (Down) truncated Naca0009 hydrofoil

\section{Vibration Measurements}

A piezoelectric accelerometer (Kistler 8702B25M1) is fitted in the profile support in order to monitor the mechanical response to the vortex shedding. According to spectral analysis and sudden increases of vibration amplitude, hydro-elastic couplings are detected and their hydrodynamic conditions quantified.

The portable digital vibrometer (Polytec PDV100) is used to perform non intrusive vibration measurement at different locations of the hydrofoil as shown in Figure 2. Its measurement principle is based on the detection of the frequency shift of the laser beam reflected on the profile surface (Doppler phenomenon). The frequency shift is directly related to the displacement velocity of the profile in the laser direction.

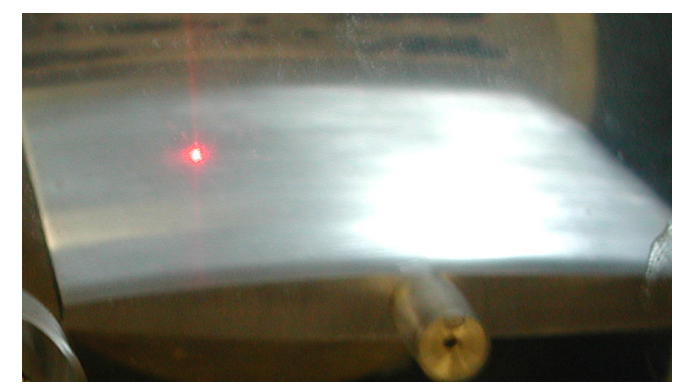

Figure 2 : Portable digital vibrometer setup for hydrofoil vibration measurements

The data acquisition system is composed of HP 1432A digitizers fitted in a VXI crate. Each digitizer has 16 input channels, 16 bits A/D resolution, a memory depth of 1 Mega samples/channel and a maximum frequency of $51.2 \mathrm{KHz}$.

\section{Double laser optical device}

A specific optical device (see Figure 3) is developed to investigate the cavitating vortex street. Two laser beams are set to cross the wake and their light intensities are measured with the help of fast photodiodes. The corresponding signals are related to the vortex shedding frequency. In addition, the vortices advection velocity is deduced from the crosscorrelation of the signals and the distance between the two laser beams $(4 \mathrm{~mm})$. The device being mobile in the chord length direction, the advection velocity can be measured at different locations.

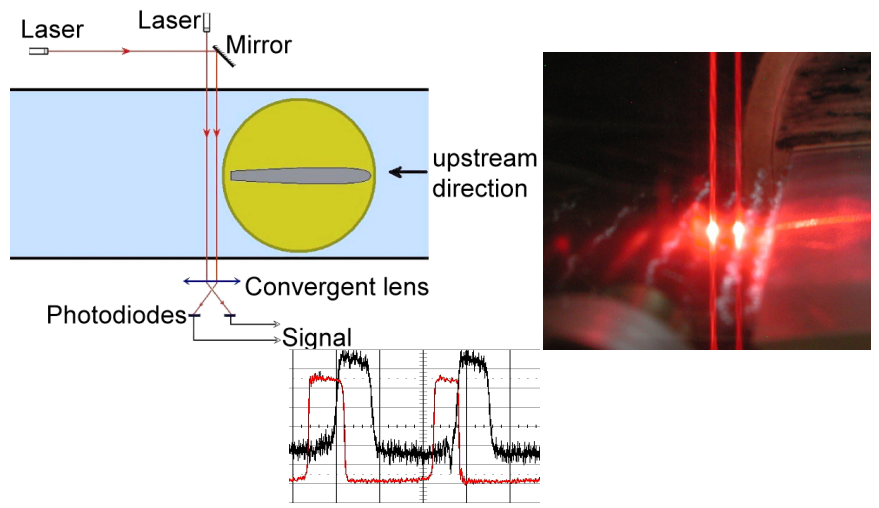

Figure 3 : Double laser optical device 


\section{Flow Visualisation}

High speed visualisation is achieved with the help of the Weinberger Visario 1500 video camera. The CCD image resolution is $512 \times 192$ pixels at the highest rate $\left(10^{\prime} 000\right.$ frames/sec). An electronic shutter allows the setting of the exposure time down to $10 \mu$ s. The light source is provided by two Cordin Xenon lamps with a minimum flash duration of $11 \mathrm{~ms}$. Visualisations are recorded for different hydrodynamic conditions.

Photographs are also performed with the help of a short duration xenon flash lamp.

\section{FLUID-STRUCTURE INTERACTION IN CAVITATION FREE REGIME}

The modal analysis of the tested hydrofoil has been performed by Dupont et. al. [2]. In water, the main vibration modes have been identified as the first and second bending modes as well as the torsional mode, corresponding to eigen frequencies of 700,1950 and $900 \mathrm{~Hz}$ respectively.

The mechanical response of the profile to the shedding of von Karman vortices in cavitation free regime is monitored with the help of the portable digital vibrometer and the accelerometer. Through spectral analysis, the vortex shedding frequencies are obtained and the hydrodynamic conditions at which hydro-elastic couplings take place are deducted. To this end, the upstream velocity is increased/decreased $(5-30 \mathrm{~m} / \mathrm{s})$ and signal acquisitions are performed for every velocity step. The static pressure at the inlet of the test section is maintained constant to preserve the wall deformations and consequently the boundary conditions of the profile. Moreover, this pressure is chosen high enough to ensure cavitation free flow in the whole velocity range ( $p_{\text {inlet }}=4.75$ bar).

Considering the waterfall spectra of the portable digital vibrometer signals (Figure $4 \mathrm{Up}$ ), the linear relationship between shedding frequency and upstream velocity is observed and found to be in accordance with the Strouhal relation (mean value $\overline{S t}=0.236$ ). As the flow velocity is increased/decreased so that the shedding frequency approaches one of the natural frequencies of the combined fluid-structure system, a hydroelastic coupling occurs and significant increase of vibration level is observed. The mentioned linearity is no more observed for upstream velocity ranging from 11 to $13 \mathrm{~m} / \mathrm{s}$, where the shedding frequencies remain constant. This is due to the lock-in phenomenon corresponding to the second mode of vibration of the profile $(900 \mathrm{~Hz}$, torsional mode). In view of the vibration amplitude, three other hydro-elastic couplings are also detected and occur for the following upstream velocities: 7, 9 and 28 to $29 \mathrm{~m} / \mathrm{s}$. In Figure 4 (Down), the predominant frequencies of the accelerometer and portable digital vibrometer signals are represented as function of the upstream velocity. The Strouhal linearity and the frequency lock-in are highlighted by both measurement device signals. Moreover, the presented evolutions are in perfect accordance one with the other.

\section{VISUALISATION}

The cavitation that develops in the core of the Karman vortices allows a visualisation of the vortex street. Although this mean of visualisation may influence the dynamic of the vortex street [2], it is believed that the original wake geometry and morphology are preserved at early stage of cavitation.
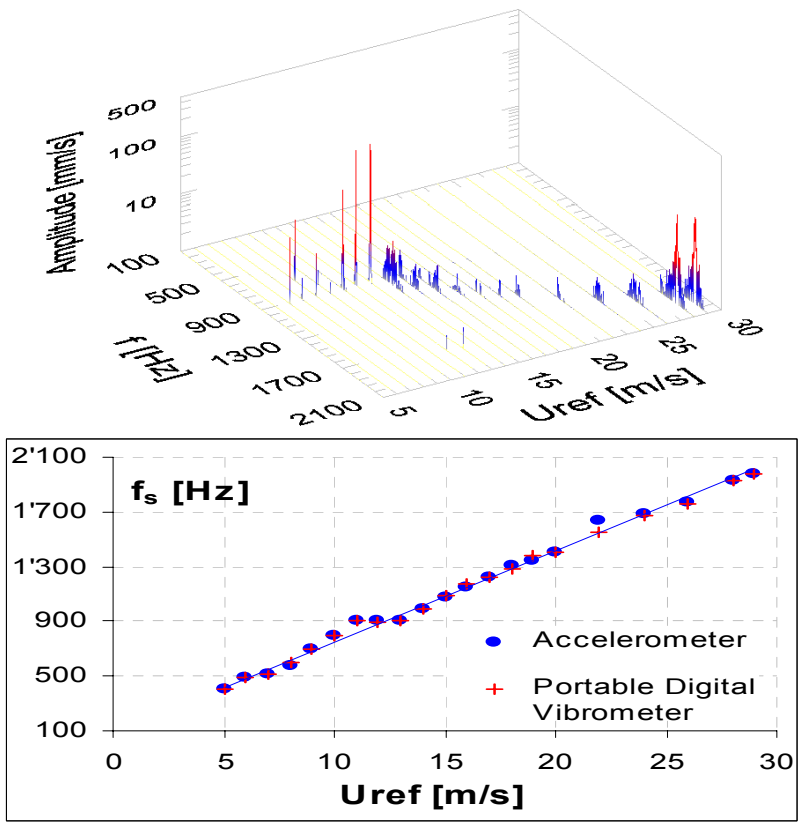

Figure 4 : (Up) Waterfall spectra of the portable digital vibrometer signals (Down) Shedding frequency of Karman vortices for different upstream velocities

Figure 5 presents two series of top-view photographs of cavitating von Karman vortex street. It is found that when the hydrofoil is not resonating, Figure 5 (a), the wake exhibits 3D instabilities despite the fact that the hydrofoil is 2D. Strong distortion and random spatial evolution of the vortical structures is observed. Such hydrodynamic instabilities result from a complex fluid-structure interaction. In fact, the vortex intensity is highly influenced by the micro-displacement of the hydrofoil trailing edge. According to Karman theory on the stability of the vortex street, the advection velocity, which is related to the vortex intensity, is consequently influenced by the vibration of the hydrofoil trailing edge. In the case of lock-in, Figure 5 (b), the trailing edge transverse vibration leads to more organized wake and a 2D vortex street is observed. It should be noticed that the oscillation amplitude of trailing edge displacement is around $0.4 \mathrm{~mm}$ under lock-in conditions.

We have presented in Figure 6 the result of high speed visualisation of the Karman street in the case of hydro-elastic coupling (torsional mode, $U_{r e f}=12 \mathrm{~m} / \mathrm{s}$ ) for two values of cavitation index $\left(\sigma / \sigma_{i}=0.85\right.$ and 0.7$)$. The five frames represent one generation cycle. We may observe that during the motion of the trailing edge from its minimum to maximum transverse location, the fluid in the recirculation zone starts rolling-up without being advected by the mean flow. Due to pressure level, cavitation occurs in the core of the vortex and starts growing. When the hydrofoil reaches its maximum transverse location, it starts its backward motion. It is precisely the time at which the vortex detaches from the trailing edge and accelerates in the hydrofoil wake. The lower vortex is generated in a similar way during the backward motion of the hydrofoil. 

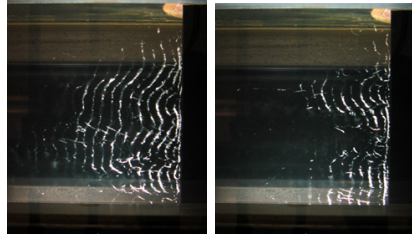

(a)
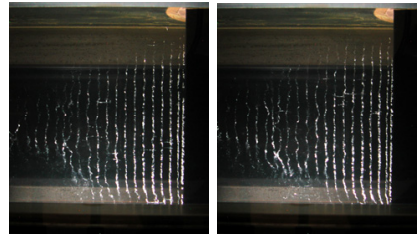

(b)
Figure 5 : Time series of top-view photographs of cavitating von Karman vortex street (flow from right to left)

(a) Lock-off condition $\left(U_{\text {ref }}=25 \mathrm{~m} / \mathrm{s}, \sigma=0.5\right)$

(b) Lock-in condition, torsional mode $\left(U_{r e f}=12 \mathrm{~m} / \mathrm{s}, \sigma=0.7\right)$
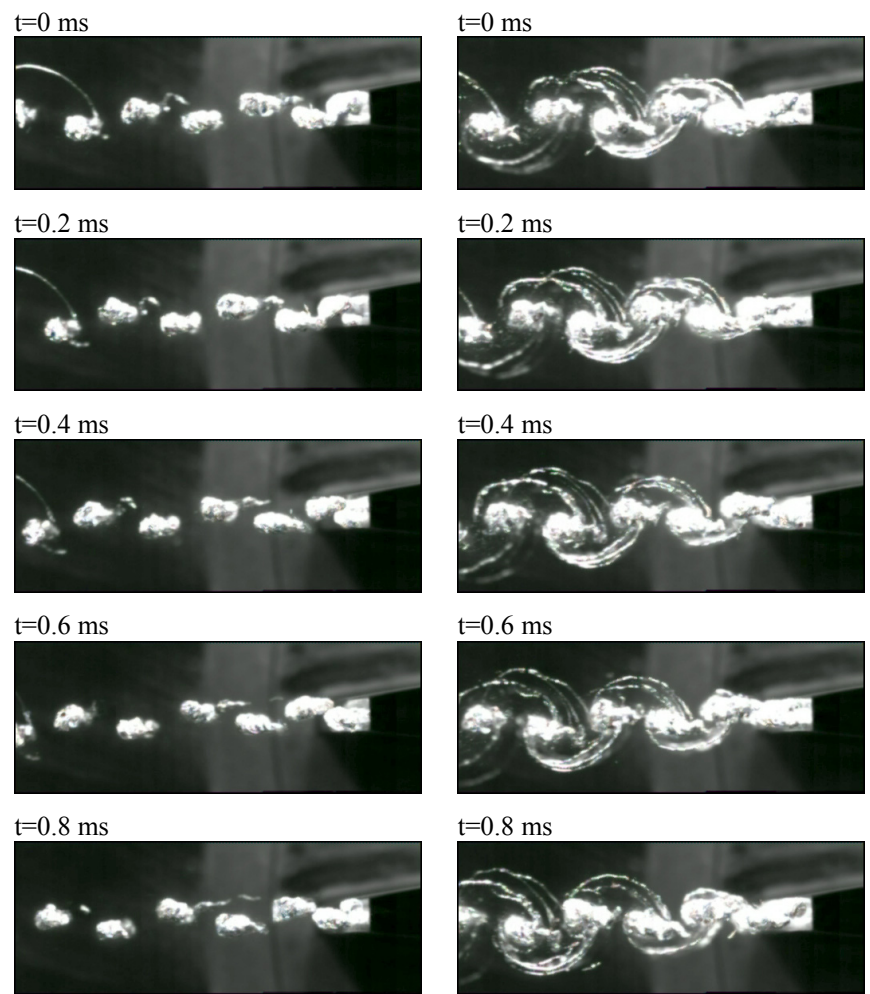

(a)
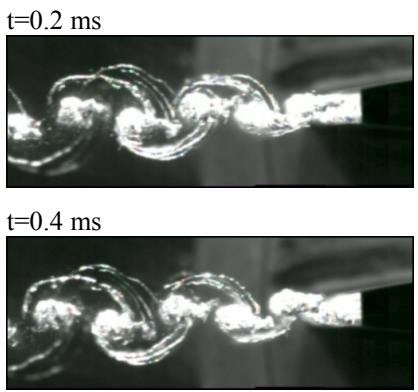

$\mathrm{t}=0.6 \mathrm{~ms}$

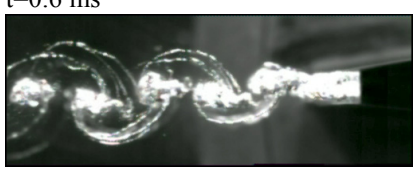

$\mathrm{t}=0.8 \mathrm{~ms}$

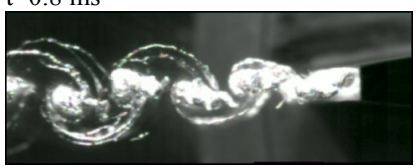

(b)

Figure 6 : Five frame series of von Karman vortex street for lock-in condition $\left(U_{r e f}=12 \mathrm{~m} / \mathrm{s}\right)$ and for two cavitation levels, flow from right to left: (a) $\sigma / \sigma_{i}=0.85$, (b) $\sigma / \sigma_{i}=0.7$

\section{CAVITATION EFFECTS ON VON KARMAN VORTICES}

\section{Cavitation inception}

Models for cavitation inception in the wake of bluff bodies have been presented among others by Belahadji et al. [6], Arndt [8] and Pauchet et al [9]. They all lead to $\sigma_{i} \sim \sqrt{\mathrm{Re}_{\text {chord }}}$ law, which correctly predicts the correlation between the incipient cavitation index and the Reynolds number for the considered range of upstream velocities. In our case study, the incipient cavitation index is presented in Figure 7 as function of the root square of the Reynolds number. The cavitation inception is detected visually.

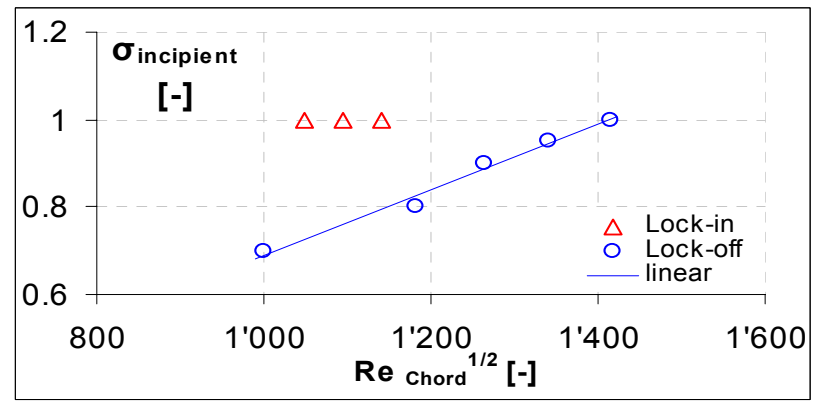

Figure 7 : Von Karman vortices incipient cavitation number for different Reynolds conditions

In lock-off condition and according to the linear tendency curve in Figure 7, the relation between the incipient cavitation index and the Reynolds number can be formulated as $\sigma_{i} \sim 0.0007 \sqrt{\mathrm{Re}_{\text {chord }}}$.

The incipient cavitation index in the case of lock-in conditions (torsional mode) is much higher than the one in lock-off cases. The vibration amplitude of the profile generates additional vorticity which lead to an early cavitation inception.

\section{Vortex shedding frequency}

The cavitation influence on the vortex shedding frequency is studied for three different upstream velocities at 18,16 , and $12 \mathrm{~m} / \mathrm{s}$, the last one conditioning the lock-in. Measurements are conducted using the accelerometer, the portable digital vibrometer and the double laser optical device. Through spectral analysis of the different signals, the resulted main frequencies are in perfect agreement. The vortex shedding frequencies detected from the accelerometer signals are presented in Figure 8 (Down) as a function of the cavitation degree. The shedding frequency $f_{s}$ is normalized with its corresponding value at cavitation free regime $f_{s(\sigma>\sigma i)}$. Waterfall spectra are presented in Figure 8 (Up) for an upstream velocity of $16 \mathrm{~m} / \mathrm{s}$. As soon as the cavitation appears in the core of Karman vortices, a significant increase of the vibration amplitude and the shedding frequency is observed. As the cavitation index is further reduced, the shedding frequency keeps increasing in a almost linear way. When the cavitation is fully developed $\left(\sigma / \sigma_{i}<0.4\right)$, the vibration amplitude falls as low as in cavitation free regime. In this case, the shedding frequency is no more meaningful since the vortex structures merge to form a single vapour cavity.

The cavitation effect on von Karman shedding frequencies is presented in Figure 8 (Down) in lock-in and lock-off conditions. A notable influence of the cavitation is observed out of lock-in at 16 and $18 \mathrm{~m} / \mathrm{s}$. As soon as the cavitation appears in the wake $\left(\sigma / \sigma_{i}=1\right)$, a sudden frequency increase $(3 \%)$ occurs. Out of hydro-elastic coupling condition and when cavitation develops, the shedding frequency values increase as high as $15 \%$ more than the value in the non-cavitating regime. Beyond a certain volume of vapour, the shedding frequency falls off. These frequency variations towards the cavitation index are consistent with the ones reported in prior studies on the wake of symmetric wedges ([5], [6]). 

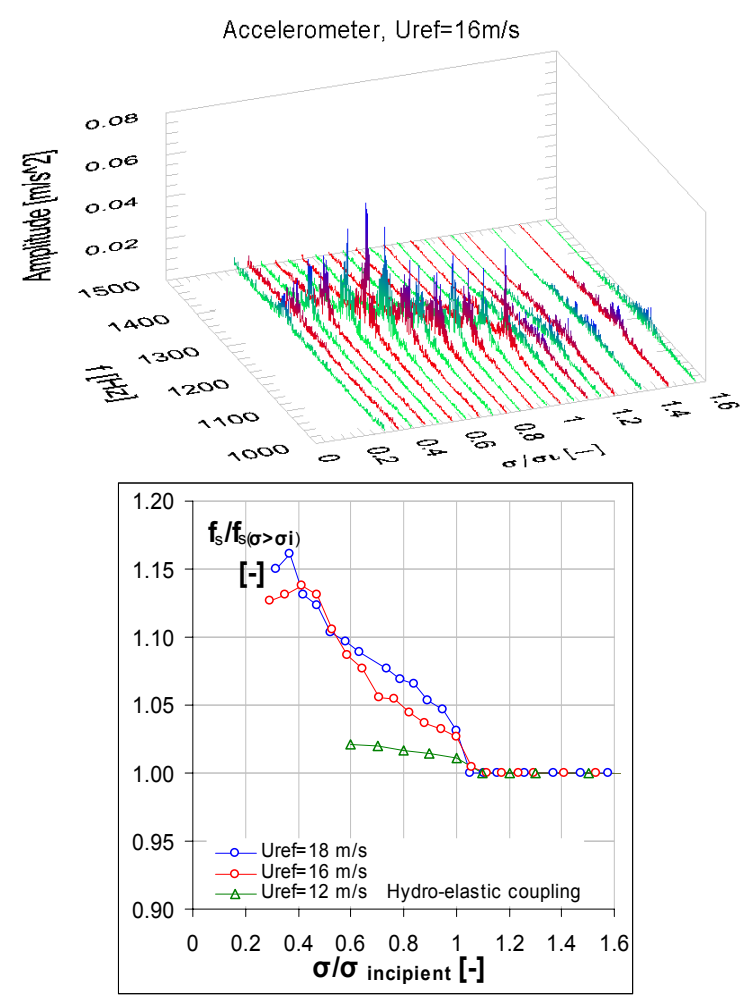

Figure $8:(\mathrm{Up})$ Waterfall spectra of the acceleration signals for different values of cavitation index at $16 \mathrm{~m} / \mathrm{s}$ upstream velocity (Down) Shedding frequency of Karman vortices vs cavitation index for different upstream velocities
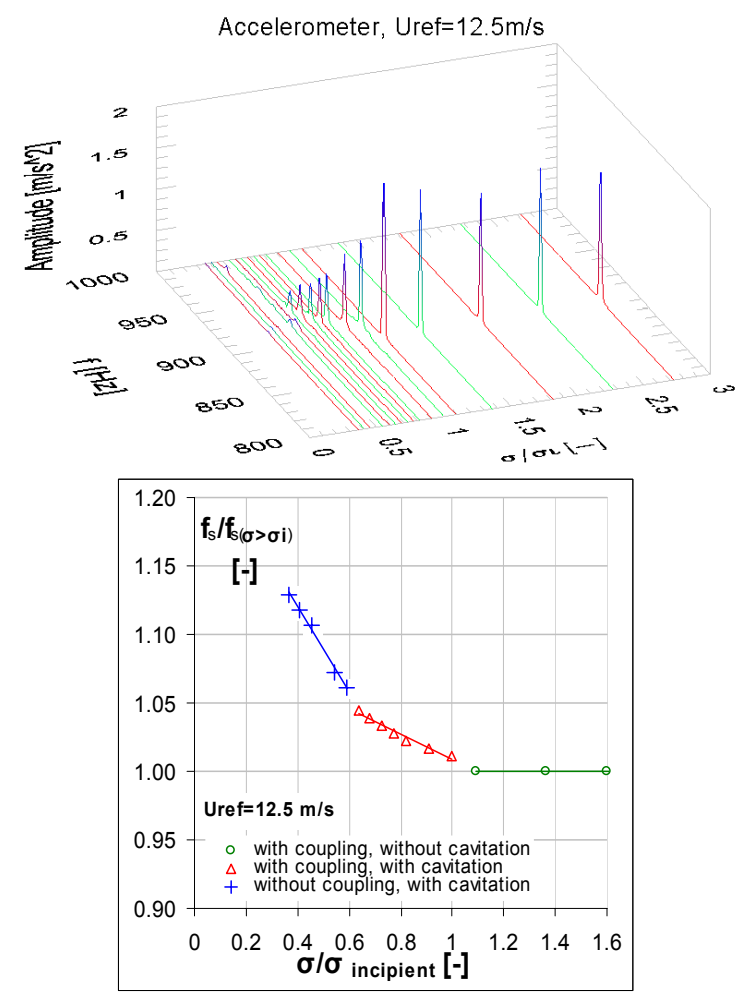

Figure 9 : (Up) Waterfall spectra of the acceleration signals for different values of cavitation index at $12.5 \mathrm{~m} / \mathrm{s}$ upstream velocity (Down) Shedding frequency of Karman vortices vs cavitation index at $12.5 \mathrm{~m} / \mathrm{s}$ upstream velocity
In the case of hydro-elastic coupling condition at $12 \mathrm{~m} / \mathrm{s}$ and as soon as the cavitation appears in the wake, a slight increase in frequency occurs for decreasing values of the cavitation index. But this increase remains definitely lower than the one recorded without resonance. The profile vibrating at or near its eigen frequency, it imposes the vortices to shed at this frequency. Nevertheless, considering these frequency increases, we found that the hydrofoil resonance may be whether avoided (Figure 9) or triggered by cavitation development (upstream velocity maintained constant).

In Figure 9, a constant shedding frequency is obviously observed for the non cavitating state $\left(\sigma / \sigma_{i}>1\right)$, the frequency being entirely determined by the upstream velocity. As soon as the cavitation appears in the wake and with its development, the resonance is maintained whereas the vortex shedding frequency increases up to $5 \%$. For further decrease of the cavitation index, the hydro-elastic coupling is no more maintained so that the vortex shedding frequency increases in a more marked way.

\section{Advection velocity of the vortex street}

The double laser optical device allows the measurement of the vortices advection velocity in different locations. The reduced velocity $U^{*}$ is presented in Figure 10 as a function of the normalized distance from trailing edge $d / c$. The cavitation degree and the upstream velocity are maintained constant at $0.6[-]$ and $12.5 \mathrm{~m} / \mathrm{s}$ respectively so that the lock-in exists. We note an increase of the advection velocity in the very near wake $(d / c<0.15)$, this zone being the place of the vortices generation. For longer distances, a quasi-constant reduced velocity $U^{*}$ is reached of about 0.9 .

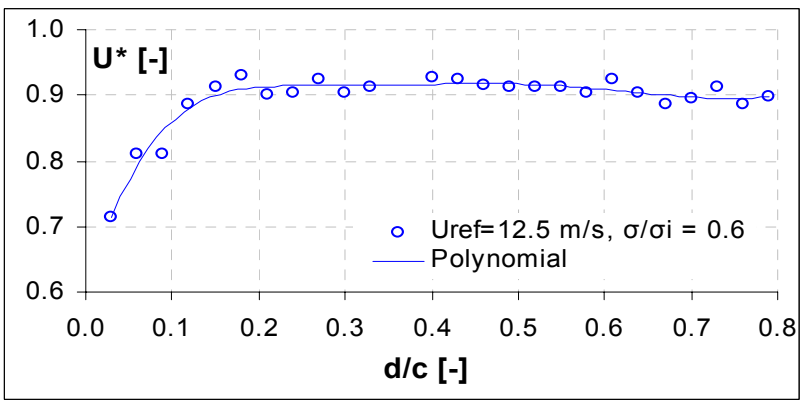

Figure 10 : Reduced advection velocity of the vortices for different distances from trailing edge

The reduced advection velocity of the vortices for different cavitation degrees is presented in Figure 11. The upstream velocity is maintained constant $\left(U_{r e f}=15 \mathrm{~m} / \mathrm{s}\right)$ and the measurements are performed at a distance from the trailing edge of $20 \mathrm{~mm}(d / c=0.2)$. We observe an increase of the vortices advection velocity with the development of the cavitation in their core ( $4 \%$ for $\Delta \sigma=0.4)$. The increase is linear and occurs as soon as the cavitation appears in the wake. 


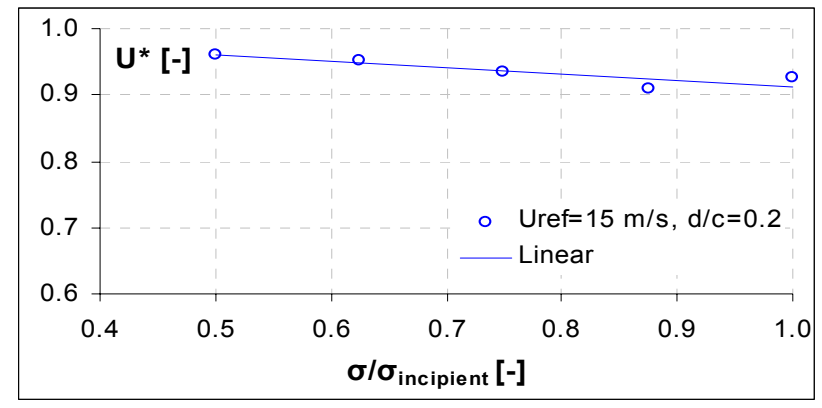

Figure 11 : Reduced advection velocity of the vortices for different cavitation degrees

\section{Streamwise spacing of vortices}

High speed visualisation can be carried out with the aim of measuring the distance between the vortices. But phase averaging is meaningless due to the distortion of the vortex street and its 3D evolution. To avoid this practical difficulty, we calculate the streamwise spacing using the measurements of the vortices advection velocity and their shedding frequency. The streamwise spacing $A$ is deduced from the following statement:

$A(t)=x(t+T)-x(t)=\int_{t}^{t+T} U_{\text {Karman }}(\tau) d \tau$

where $x(t)$ is the position of a vortex at time $t$ and $T$ the period of the shedding process. The evolution is represented in Figure 12 with the streamwise spacing $A$ tending to slightly decrease for decreasing cavitation index.

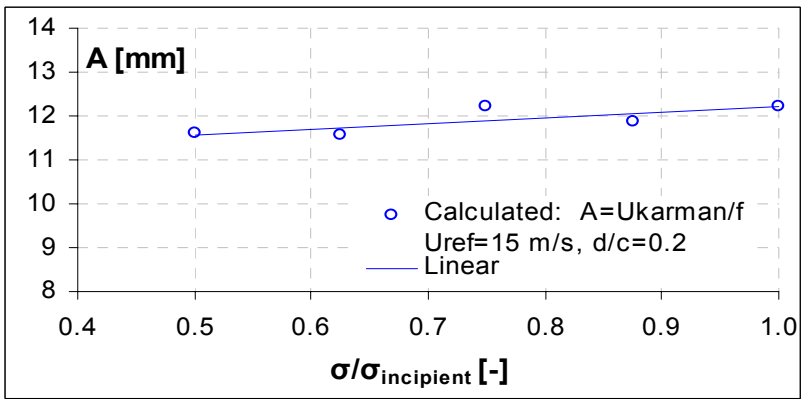

Figure 12 : Streamwise spacing of vortices in the same row vs cavitation degree

\section{CONCLUSION}

In the present study, we have performed an experimental investigation on the fluid-structure interaction caused by Karman vortices in the wake of a truncated 2D hydrofoil. In addition to measurements of structural vibration in the support of the profile, a portable laser vibrometer is used. Moreover, a specific optical device is developed to investigate the dynamic of the cavitating wake. Finally, the generation mechanism of Karman vortices has been investigated with high speed visualisation. The main results of the current study are summarized as follows:

- The survey of the generation frequency of the Karman vortices with respect to the flow velocity reveals the Strouhal behaviour. The mentioned linearity is no more observed for upstream velocities ranging from 11 to $13 \mathrm{~m} / \mathrm{s}$, where the shedding frequencies remain constant. This is due to the lock-in phenomenon corresponding to the torsional mode of the profile. In view of the vibration amplitude, three hydro-elastic couplings are also detected.

- The cavitation that develops in the core of Karman vortices allows a visualisation of their morphology and dynamic. It is found that when the hydrofoil is not resonating, the wake exhibits 3D instabilities despite the fact that the hydrofoil is 2D. Strong distortion and random spatial evolution is observed. In the case of lock-in, the trailing edge transverse vibration organizes the wake and a $2 \mathrm{D}$ vortex street is observed.

- The cavitation occurrence in the wake leads to a significant increase of the hydrofoil vibration amplitude and the vortex shedding frequency. This generation frequency increase up to $15 \%$ in lock-off conditions but significantly lower in lock-in. It is also observed that hydrofoil resonance may be avoided or triggered by cavitation development.

- The study of the Karman vortices dynamic reveals that their advection velocity increases about 4\% with the development of the wake cavitation meanwhile the streamwise spacing is reduced.

\section{ACKNOWLEDGMENTS}

The presented research has been performed in the frame of the HydroDyna project. All industrial partners are thanked for their contribution. The CTI Innovation Promotion Agency are also thanked for their financial support.

\section{REFERENCES}

[1] Avellan F., Henry P., Ryhming I.L, (1987) "A new high speed cavitation tunnel”, ASME Winter Annual Meeting, Boston (USA), FED-Vol 57, pp. 49-60

[2] Dupont Ph., Avellan F., Wegner M., 1987, Wake flow analysis for a hydrofoil with and without hydroelastic lock-in, Proc. of the Int. Conf. on Flow Induced Vibration, UK, 12-14 May, 1987, pp. 417-426

[3] Rockwell D., 1998, Vortex-body interactions, Annual Reviews in Fluid Mechanics, 30, p199-229

[4] Williamson C.H.K, Govardhan R., 2004, Vortexinduced vibrations, Annual Reviews in Fluid Mechanics, 36, p413-455

[5] Young J.O., Holl J.W, March 1966, Effects of cavitation on periodic wakes behind symmetric wedges, J. of basic engineering, p163-176

[6] Belahadji B., Franc J.P., Michel J.M., Cavitation in the rotational structures of a turbulent wake, J. of fluid mechanics

[7] Williamson C.H.K, Roshko A., 1998, Vortex formation in the wake of an oscillating cylinder, J. of Fluids and Structures, 2, p355-381

[8] Arndt R.E.A, 1976, Semi-empirical analysis of cavitation in the wake of a sharp-edged disk, J. of fluids mechanics, p560-562

[9] Pauchet J., Retailleau A., Woillez J., 1992, The prediction of cavitation inception in turbulent water jets, Cavitation and Multiphase Flow Forum, SED, 135, p149-158 